\title{
VI. PANEL DISCUSSION OF FUTURE RESEARCH
}




\title{
PANEL DISCUSSION OF FUTURE RESEARCH
}

\author{
Panelists: G.J.Peters \\ A.G. Hearn \\ H.F. Henrichs \\ T. Kogure \\ C.T. Bolton \\ M.A. Smith \\ Moderator: M. Plavec
}

Plavec:

Some time ago Arne Slettebak approached me with the idea that this colloquium should be properly concluded by saying what should be done in the future with Be stars, and I immediately volunteered to do the job. I proposed that I would just stand up and say in the future someone should at last explain the Be stars correctly and completely and that would be the end of the study, but Arne rejected this idea completely. He said that this imaginative and original and bold idea would shock the people so much that there could be terrible consequences. He said he does not want to have any fatalities at the end of the colloquium, and he added that he likes me, so I'm not quite sure who he thought the fatality would be. He also maintained that great truths should be delivered to people in small doses, like antibiotics, and therefore a panel of several people has been selected to administer the small doses of great truths to you now during this session. The idea is as follows: Each of the panelists will speak for 5 minutes. Remember please, do not interrupt them, this is their only opportunity instead of an invited talk to say what they think should be done in the future. I hope each of them will focus on only 1 or 2 narrow topics, maintaining what is very important for the future to accomplish in our field. Then their comments will be open for discussion, but please, the discussions should consist only of short comments violently agreeing with and augmenting their argument or violently disagreeing with and criticizing their approach as absolutely impossible and really nowhere. So, just essential comments on what they have said; no extension into a different field because it may be picked up by someone else on the panel. I expect that each discussion will not last longer than 7 minutes, and then the next panelist will speak. I am afraid there will be practically no time left afterwards, but if there is time left, we will find out what to do. We will start with the only lady of the panel which is G.J. Peters.

Peters:

As I see it, we can divide goals in Be star astronomy into two broad categories. We can search for a cause of the Be star phenomenon, or we can model the circumstellar matter that presently exists and assess and determine the nature of its variability. Included in the latter category are research projects aimed at determining the geometry, the sizes, the physical conditions, flows, etc. in existing Be envelopes or circumstellar matter. To research the cause of the Be phenomenon, I think it is important to observe the stars during quiescence. The idea here is that if we want to find out the cause of the illness we have to observe the stars while the illness is developing. After the disease is in place all we can do is treat the symptoms, so I think that what we have to do is obtain more observations of Be stars during their interesting quiescent phase.

I have enumerated five research projects or goals that especially interest me 
at this time. First, I think we should investigate the nature of the long-term variations in the far ultraviolet flux. Those variations involve a few hundred solar luminosities, so it is not a minor amount of energy that is involved. Second, I am interested in correlations between $V / R$ in the Balmer lines and variability in other spectral features. In connection with this, I wonder whether the Paschen, Brackett, and Pfund lines also show the same $V / R$ variations. Are they optically thick or thin? I think that now we are capable of getting observations in the 2 micron region, and these observations will reveal a lot about the nature of the cool circumstellar matter in Be stars. Third, I think we should try to find some radial velocity curves for the interacting binary Be stars. I will talk about that more in a little bit. Fourth, I think we should investigate transient phenomena and determine what the precursors of these transients are and how they are related to overall activity. Fifth and finally, we need to further investigate how Be and B stars may differ.

For most of these projects long-term observations are in order, and for many of them simultaneous observations are necessary, in particular for the determination of the reasons for the long-term far- UV variability, which involves several hundred solar luminosities. From our Voyager data we observe a flux change. Determining whether it is due to a change in the effective temperature or a change in the redistribution or some other cause will require simultaneous multi- frequency observations (I am emphasizing more photometric observations) over a wide range. In particular I think it's important to find out what is happening in the near ultraviolet, which might receive most of the flux if it is simply redistributed.

Are $\mathrm{Be}$ stars interacting binaries according to the definition stated at the beginning of the week? The following classes of objects can be called Be stars: the "classical" Be stars (probably what most of the audience thinks of as Be stars), the interacting binaries (the Algols and $x$-ray binaries), and the pre-main sequence stars. We can easily separate these out in some instances because of other observable characteristics. I want to emphasize, however, that in many cases it is not easy to tell which stars are interacting binaries. Determining the radial velocity curves is not easy. I want to emphasize that for the periods (50 to 150 days) and the mass ratios (only 0.1 to 0.3 ) that we expect to exist in these interacting binary Be stars, most of the expected radial velocity variations are less than 15 $\mathrm{km} / \mathrm{s}$ for semi- amplitude. Measuring the centroids of complex profiles to that accuracy is a very tricky business.

In order to aid in identifying the Be stars which acquire their circumstellar envelopes through binary mass transfer, I recommend that observers do not automatically eliminate from their programs stars which are known to be interacting binaries. We need to find out how the spectroscopic and photometric behavior of these stars compares with that of single stars. Alternatively, I urge the binary star community to study the interacting binary Be stars and to analyze their data with standard binary star techniques. Perhaps, by combining the talent in the two communities, we can finally determine how many interacting binaries exist among the group of "classical" Be stars.

Mendoza:

I presented a way of using spectra, say MK type of low- dispersion spectra, of the order of $100 \AA / \mathrm{mm}$, to distinguish Be stars from other $\mathrm{B}$ emission-line objects. We can distinguish between supergiant stars, we can distinguish what we have called classical Be stars, and we can distinguish some peculiarities. But it is not clear how to distinguish 
Herbig Be stars just from the spectroscopic point of view without knowing the location of the stars in the H-R diagram. In regard to spectra, except in a few cases where the emission is extremely strong (we may suspect that these are Herbig Be stars), I think it would be nice if somebody would undertake the job of studying very carefully the spectral classification of Be stars to include all types.

Peters:

So what you are saying is that a number of pre-main sequence objects may masquerade as classical Be stars?

Mendoza:

Yes, you can't distinguish them from the spectroscopic point of view.

van den Heuvel:

One of the most exciting things I heard about in this conference is this far UV variability of large amounts, say 100 solar luminosities. I may have said this already, but I think that almost 20 years ago Paczynski, Plavec and Kippenhahn carried out calculations of interacting binaries showing that in many cases you expect a helium star to sit next to a main sequence star after the mass transfer. This helium star is extremely hot and has a very large far ultraviolet flux. I wonder whether the large far-UV variability in Be stars may have something to do with those helium stars, which nobody has found so far, I think, in the intermediate-mass cases. In the high-mass cases, many people have suggested that those stars are Wolf-Rayet stars, but other people don't agree with that. But I think in the intermediate-mass case nobody has ever found a companion of this helium type, and those stars must have a hundred to thousand solar luminosities in the far ultraviolet.

Peters:

Are you suggesting that the helium star itself varies?

van den Heuvel:

Those stars are very compact and have very large radiation fluxes. I could imagine that they do strange things. They are probably a bag of photons of $\gamma=4 / 3$, so they probably are variable.

Peters:

We have, in a preliminary way, fit Kurucz models to the energy distributions, and they are consistent with what you would expect for the spectral types of the stars. In other words, we don't see any obvious evidence for a very high-temperature component. But that is a very interesting idea.

Baade:

I think most of us are prepared now to admit that not a single model will explain all Be stars. I think these known Be binaries are very helpful in starting to sort out the various possible populations, and it seems rather certain now that there is a substantial difference in the line profile variability between the Be and the Bn stars. I think one should also look for similar differences between the possibly Be stars and these known Be binaries, to see if there is another difference between these two populations. 
Peters:

Indeed, we have been pursuing that problem.

Percy:

A very brief comment: Let's not forget that there are often some normal B stars that show these same phenomena such as, I understand, flux variations in the far ultraviolet.

\section{Doazan:}

I want to comment on the comment of van den Heuvel, because the suggestion that it's a compact object that undergoes temperature variations is contradicted by the 88 Herculis observation, which shows the association between the drop in temperature and the appearance of a shell phase.

Peters:

Since you brought up 88 Herculis, I would like to show the light curve of $\mathrm{AU}$ Mon. This is an acknowledged eclipsing interacting binary Be star, and when the ultraviolet shell features are present, the star is faint and behaves in exactly the same sense as 88 Herculis.

Plavec:

We must stop here; if perhaps you have more comments, maybe you can make them later. Now, our next panelist, Dr. Hearn.

Hearn:

Unlike most people here, I have never worked on Be stars so I can be considered as a theoretician who knows very little about the subject. However, having followed Be stars in a general sort of way, I would like to summarize observations that on a global scale I think are important from the theoretical point of view. At the top of the list I have put variability on all sorts of timescales. Mostly there is no defined periodicity. There is just random behavior and that random behavior is something I want to come back to.

We have heard at this conference of infrared and hard x-ray observations that are interpreted in terms of disks. We have heard of observations which are interpreted in terms of non-radial pulsations. We have heard about observations on polarization. We have heard interesting remarks on the relation between narrow absorption components and Be stars, and the way Be stars are traditionally seen to be fast rotators. There doesn't really seem to be in many cases much correlation between vsini and the observations, although I think there is something in the argument that there is a threshold.

In terms of future work, I think the developments in non-radial pulsations are very interesting, and we certainly have a need for more theory in that area, particularly in terms of the excitation mechanisms of non-radial pulsations, and I think we need a further exploration of the physics of disk models. What I really want to lay my emphasis on is the way in which Be stars vary with time. I think if we are going to understand this, then we should look away from Be stars to other fields of physics and a field of mathematics which is becoming increasing important, and that is the theory of non-linear equations. This is a field which is developing very rapidly. Very simple non- linear equations can 
have very complicated properties. For example, you can have a whole range of solutions where as you increase a driving force, you go from a stationary solution to a one-period relaxation oscillation and then you get other periods coming in very quickly with irrational ratios of frequency. There is no frequency doubling, which comes in quite irrationally, and then very quickly all these non-linear irrational periods combine to give you what is called chaos. It is chaos in the sense that it is completely non-deterministic that because of the nature of the equations you cannot predict what is going to happen for very long ahead. A good example of this is the weather, and we see this week how that can be unpredictable. Another physical example is the way that turbulence develops as a result of flow against an obstacle. If the velocity is slow, one comes to a streamline, stationary solution. Then you get vortices breaking off one at a time with irregular period, and that is a one-period relaxation oscillation, and then if you go much higher it rapidly breaks away into turbulence. Another feature of non-linear solutions is that one gets quasi-periodic solutions. One can see something like a period, but there is no really defined period. That is something we have heard a number of times in this meeting; that something varies with time, that there is some sort of characteristic time, but you look at it closely and there is no well defined period. This is quite typical of another regime of non-linear equations. The sunspot cycle is a very good example of that. You have a 12-year cycle. It is not exact, and sometimes it just disappears as it did in the Maunder Minimum. One sees similar examples of this in the light curves of Miras. If you look at 40 years worth of Mira light curves, you see that each cycle is similar to the last, but it's not exactly the same. The period varies slightly, the amplitude varies slightly, the form varies slightly, and sometimes it stops and starts off again in another phase.

Finally, one has bi-stable solutions that can flip from one stable solution to another. An interesting example of this is work done in Utrecht, not in the observatory, but in the Geophysics Department. Theoretical studies of the icecap of the earth show that you have a bi-stable state. One has a glaciated state and a non- glaciated state, and if you have a thousand earths and you observe them, then observers would say we have two different types of earth. We have one that is glaciated and one that is not glaciated. But, that is not true. You have an earth which is non-linear. It's a result of this non-linearity that one gets a time-varying solution even though all the physical inputs are constant. So, even though you have a constant luminosity, a constant effective temperature, constant physics, you can still get things that are varying with time in a random period with no obvious correlation, and that is what we have in Be stars.

I would like to finish with a heartfelt plea as a theoretician to all the observers here. Please remember that nature is much more complicated than theoreticians are, and one has a simple theory that can only deal with global qualities. Please do not reject a simple theory because it doesn't fit in with your favorite star or your favorite observation. This summer I took my holiday in Scotland, and we passed through one of the old oak forests that one finds up there. I am sure that a theoretician dealing in forests could explain why that oak forest was there:, temperature, climate, soil, mountainous elevation, and everything else. A iairly simple theory. Then an observer would come along, walk through the woods and say, "Very interesting: this tree is an ash. Does your theory explain why that ash is present?" This is a case of not being able to see the wood for the trees. Because no one here is an expert on trees, everybody understands what I'm talking about. But, I am afraid now we're going to get to Be stars, and that clarity of vision is going to 
disappear instantaneously.

Stalio:

I am referring to your comment that the infrared gives you information only on disks, and that the infrared data you referred to in the review paper favors the disk model. In my opinion, without offending anybody, especially the speaker, the infrared data can also be explained by spheroidal or spherical models.

Hearn:

I chose my words very carefully. I said that there are observations which have been interpreted in terms of disks. Let me make this point: that Henny Lamers gave an extremely lucid talk, where he explained how different parts of the observations can be fitted with the disk model. The disk model is completely arbitrary in the sense that it is not based on a physical model, but one has to start with this when one does the observations. I also hear from Rens Waters and Henny Lamers that they have not been able to fit a spherically symmetric model. I have discussed this at various times, and I think they have made an honest attempt to make that fit, and they haven't done it. I am susceptible to lucid expositions where one sees how a model fits, and up until now I haven't seen that done for the spherically symmetric models any more than I have seen it done for $\mathrm{H} \alpha$ observations. I hope that perhaps at the next Be conference this interpretation will be produced in the same sort of lucid style as Henny did.

\section{Harmanec:}

In general, I am quite sympathetic with your warnings concerning the stochastic processes, but my feeling is that these apply to such effects, if I go with your comparisons, as waves in the river. But I suspect that we are in the position where we just try to see the rivers on the stars, and these have some defined shape. So, we probably are not able to go into such details at the moment, and should not exaggerate these details because we are not yet in position to do so with the observations.

Hearn:

I don't think you should interpret my remarks as details like waves on a river. One has, in coronal loop physics, for example, work done by my colleagues Cowen and Maartens. Coronal loops would appear to have for 24 hours a hydrostatic stationary; a stationary structure, so that the lifetime of the loop is very much longer than any physical characteristic timescale of those loops. What they showed was through some intelligent physical approximations that one had a coronal loop which was in fact a time-dependent non-linear solution, and the whole process with time when the loop grows and disappears is the solution of the non-linear equations. What is interesting about that point is it has a long, almost constant, solution which appears to be very close to what you would get if you said coronal loops are just hydrostatic. But the whole existence and timescale of the loop is determined by the non- linear solutions. I understand what you say but I think this is not correct.

Pesnell:

I have a comment on the chaotic solutions to non-linear equations. I have seen theoretical light curves that greatly resemble Cepheids, but they are mathematically 
chaotic. However, if you look at them from an observational point of view, they look almost perfectly like Cepheid light curves. So it is very easy to conceal a chaotic solution to a non-linear equation in a relatively periodic-looking solution.

Underhill:

I think your emphasis on non-linear equations is interesting. I suggest that my model, with magnetic fields, gives reasonable reason to have non-linear equations.

Hearn:

I agree. There are many ways one can make things non-linear.

Hoflich:

I want only to comment on the infrared. It is possible to fit the infrared excess by a spherical model, but I did not have the time in my 5-minute presentation to show this. You could see it in my poster presentation.

Hearn:

I'll go and look.

Persi:

Concerning the infrared data, the presence of the disk is only a part of this because the infrared excess, as I have shown in the poster paper, can be fitted by assuming the presence of a spherical envelope only, based on the data from one micron up to 25 microns. So the infrared data cannot distinguish the presence or absence of a disk. This is very important.

Hearn:

I repeat my words, when I said that we have heard an interpretation of the observations in terms of the disk. I am not maintaining there is no other solution and I would be very interested to see these solutions. Unfortunately, up until now, I haven't seen them. I would very happy to look at them. They may be equally good. I am not saying that they are not.

Thomas:

Welcome to the real world! For 45 years people have been doing non-linear differential equations in all kinds of aerodynamics. If you come with me this afternoon, I would be delighted to show you numerical simulations of things like this from data done 40 years ago. All the non-LTE work is non-linear. All the stuff that everybody is doing, all these things are in the literature. Read! If you don't want to read them, it's your problem, not ours.

\section{Hearn:}

I have been reading, and that's why I thought it was worthwhile making these points here. I may say that any comments about non-linear mathematical solutions were singularly lacking in any remarks you have made today. 
Lamers:

I fully agree with the remarks of Stalio and Persi that the infrared excess does not say whether the model is spherical or flat. What I said is that, given the fact that we know that the optical light is polarized; we used a flat disk. We also have fit with a spherical model, and I have shown them. The fact that there is an observed correlation between the infrared excess and the polarization again suggests very strongly that the infrared comes from a disk.

Plavec:

OK, thank you. The next speaker, Dr. Henrichs, please.

Henrichs:

First of all, I am very grateful to the scientific organizing committee for giving me the chance to predict your future.

Suppose you have four different groups of astronomers. You ask the first one to make optical spectroscopic observations of the B stars which are non-supergiants. This group of observers identifies a class of stars which we call Be stars. This is very familiar to all of us. But now we realize that our second group of observers, asked to make observations of ultraviolet discrete absorption components, will identify essentially the same group of stars as a class. The third group of people are asked only to observe, with high signalto-noise, optical line profiles, and they classify a certain group of stars which have these slow traveling bumps which (just for convenience) I label $\mathrm{l}=2 \mathrm{NRP}$, and again they will find the same group of stars. The fourth group, based on polarization measurements, finds the same group of stars, which have rapid polarization episodes with essentially constant polarization angle. In all four cases you find the same thing, and that is I think a very interesting way of looking at the Be stars (of course very personal).

Let me illustrate just the second and fourth characteristics of Be stars. On an $\mathrm{H}-\mathrm{R}$ diagram where special symbols mark the stars with discrete absorption components, you find that all the Be stars must be identified with the special symbol. It is a unique correlation.

Regarding polarization, I refer to data on $\omega$ Orionis showing that a large increase in polarization occurred at the same time the star increased in brightness and also became redder, although in the UV it didn't do anything different from before. This is an example of the kind of observation which I would like to see.

Just reading the literature, one finds that there are correlations between the data obtained by our four different groups of observers. As far as I know, there are only three cases ( $\zeta$ Ophiuchi, $\lambda$ Eridani, and 6 Cephei) where on one hand the data were published on the $\mathrm{H} \alpha$ variability, and also on the discrete absorption components in the UV, and also on the NRP behavior, and they seem to be very definitely correlated. Now the important thing is not that they are correlated, but that there exist only three cases where the simultaneous data exist, and that in all three cases there was a clear correlation. I think that is a very promising point.

As an example, in one of the three ( $\lambda$ Eri), $\mathrm{H} \alpha$ slowly increased while at the same time a broad bump in the He I line decreased its amplitude by a factor of 2 . At the same time, the discrete absorption components in C IV drastically changed their character. 
This is a typical case, and it was not searched for; it was not planned. It just happened to be in the literature.

I want to emphasize the very promising role of non-radial pulsations for explaining the Be phenomenon. One attractive feature is the expectation that the amplitude of the oscillations increases toward the equator of the star. Another has to do with the energy of pulsation. A recent model of a 12-solar-mass zero-age main sequence star shows that the order of magnitude of the pulsational energy is $10^{44}$ erg for a slowly rotating star; if it rotates more rapidly, the energy increases. The pulsational period is 6 hours for all cases. The amplitude is $10 \mathrm{~km} / \mathrm{sec}$; very modest. The energy involved is too large to be easily ignored. So it's a very powerful mechanism; you need only a very small fraction of the energy to do things to the envelope of the star. Also, the role of rotation is very promising because there are many more modes available for a star to pulsate in if it rotates. A third interesting aspect is that in binary systems the perturbations of a companion can spin up the star, and can also excite pulsations by tidal waves.

My last conclusion, something already mentioned by Hearn, is that we need to know more about excitation mechanisms. The important point for the theory is to discover how to convert pulsation energy into kinetic energy. You need only a small fraction of it, but the efficiency, or whether it can be done at all, is very important to investigate. The observational needs are clear; what we need are simultaneous long- and short-term $\mathrm{H} \alpha$ data, UV observations, non- linear pulsation data, polarization measurements, and also infrared observations.

Regarding future work, I would strongly urge people working on the nonradial pulsations to try to test whether this interpretation is unique or not. Those who observe the UV discrete absorption components are urged to quantify their measurements, so that regardless of the model chosen, the data will be in a form so that it will be possible to make comparisons between stars and between different times for a given star.

the end.

One thing is certain: if you follow all these lines you will get a headache at

\section{Doazan:}

I would like to comment on your polarization results. If my memory is good, $\omega$ Orionis has always had emission. I see you have what you call a polarization episode. You have $\mathrm{H} \alpha$ in emission and a polarization of $0.2 \%$. What happened to the disk, what happened to the polarization at that time? And then you have an increase of $\mathrm{H} \alpha$ and you have the polarization going to $0.6 \%$. All the time $\mathrm{H} \alpha$ was in emission, the polarization was less than $0.2 \%$, probably the value of the interstellar polarization. The second point I would like to make is, why don't you speak about 59 Cygni? I like very much my correlation between $\mathrm{H} \alpha$ and C IV.

Henrichs:

Within 5 minutes you want me to predict the future of 59 Cygni? I would like to comment on these polarization episodes. This phenomenon has been seen 4 or 5 years in a row. It is a very promising star to follow. $\mathrm{H} \alpha$ is always there. 
Doazan:

to the disk?

If $\mathrm{H} \alpha$ is always there, even when there is no polarization, what has happened

Henrichs:

That is the headache that I am talking about.

Underhill:

I think your problem is more one of what physics to use and what observations to look at. So long as you're translating energy from the photosphere or deep in the photosphere, which is where those regions are you are talking about that have the excess energy, you have to get it out to the outermost part of the atmosphere where your lines are formed. As long as you are using only radiation to transfer energy from a to $b$, you can't do it because it transfers as well in any direction. I think until you pay attention to the physics of getting mechanical energy into something that will show up as spectroscopic states high in the atmosphere, you are going to get nowhere.

Henrichs:

I just want to thank you for underlining this importance.

\section{Harmanec:}

You could probably easily beat me because I have had the opportunity to see only a few line profiles, but my impression about this often-quoted correlation between the appearance of $\mathrm{H} \alpha$ emission and the so-called switching of modes is that what you are observing is just the filling of this line by emission. This different interpretation should also be considered or discussed. I would like to hear about that because it appears that as the envelope develops, you can have a kind of shielding of continuous radiation, and the profiles you show here look like that.

Henrichs:

I didn't talk about mode switching. Observers, inform me that mode switching is a rather strong word for the phenomenon. It is more that the amplitude seems to change, which basically gives a similar amount of energy. But, are you talking about the emission in the helium lines or $\mathrm{H} \alpha$ ?

Harmanec:

In $\mathrm{H} \alpha$ and in the helium lines.

Henrichs:

I think you are absolutely right. The observers should try to establish whether that is the correct interpretation or not.

Baade:

I would like to comment on the apparent discrepancy between the strengths of $\mathrm{H} \alpha$ and the amount of polarization. I think this discrepancy may only be apparent because it depends very much on where the matter is that causes polarization. So, the matter from where you get the $\mathrm{H} \alpha$ emission is rather far away from the star, whereas when 
you have a new mass loss which is initiated by whatever process the matter is close to the star. It is strongly illuminated and therefore the polarization that you get is much more strongly weighted toward this matter close to the star. I have done simultaneous spectroscopy of $\mathrm{H} \alpha$ and polarimetry with Dan Hayes, and he has seen such a polarization event, a very tiny one, in that star ( $\omega$ Ori). In the same night I see a very tiny event in the far red wing of $\mathrm{H} \alpha$, and that is what you expect if you have electron scattering in a rapidly rotating envelope close to the star. I think that is all self- consistent.

Koubsky:

I would like to make a comment from the observational point of view. I think that we should follow the scheme of identifying groups or classes of Be stars, and select some representatives of the different groups of the Be stars, and to concentrate observational efforts on these representatives. I have these groups in mind: Be binaries, classical Be stars, stars that are very active in the UV (showing the shifted discrete components), stars known best from the infrared, and so on. We should concentrate on these objects and observe them for a very long time in a very large spectral region, and then go back to the theory and compare the results. Otherwise you should call for observing hundreds of Be stars for 100 years, which is not practical.

Henrichs:

I think that it is very difficult to disagree.

Plavec:

OK, thank you very much. Now, Dr. Kogure.

Kogure:

I will confine my talk only to the physics of cool envelopes, mostly from the viewpoint of the spectroscopist. I have identified one basic problem, and also some selected problems which are of interest to me. To state the basic problem, I think we need to establish a definite plan to determine the physics of the envelopes. The present practice is to start from observations, and then hope to determine parameters of the envelopes. In order to do this, one has to propose a model. Yesterday morning Dr. Marlborough referred to models as black boxes. Indeed, modelling the envelopes of Be stars is a very difficult non-LTE problem. It is very complicated, and we are far from a complete solution. There are many factors or equations to be solved by taking account of the geometry, the velocity field, the inhomogeneities; things like that.

So, what is the future? How do we overcome this black box? My proposal involves three approaches. One is the extensive observations that many people are now undertaking. More and more accumulation of extensive observational results may yield important constraints on the models. Second, I propose a new approach to modelling the problem, by solving for the radiation field using equations that include all known factors, such as statistical equilibrium, radiative transfer, the geometry of the envelope, the velocity field, and inhomogeneities that are present. My third proposed approach is to take advantage of new observational techniques, such as interferometry, to make direct observations of the envelope sizes and perhaps kinematics. These direct observations will be an important check on the models. 
So, my conclusion is to push in the three areas I have outlined, and then to try to determine the structure of the envelopes. This is my idea on the basic problem.

There are many other problems I am interested in, but among them I would like to particularly emphasize the problem presented by phase changes between $\mathrm{B}, \mathrm{Be}$, and Be-shell phases. I want to emphasize the fact that we have to recognize the variety of envelope structures involved. There can be anything from a thin disk to a rather vertically extended spheroid. Pleione (28 Tauri) provides the best observational record of phase changes. Until 1938 Pleione was a normal B star. It then entered into its first shell phase, and then a Be- shell phase, in 1955. Since 1972 it has been in a second shell phase. From the $\mathrm{B}$ phase to the shell phase, the emission lines increased in strength. Even in the Beshell phase, the emission lines were still stronger than during the shell phases. Also the peak separation, indicating the physical extent of the envelope, shows that the envelope expanded and contracted. During the Be phase there were no shell lines, indicating the optical depth of the shell was small at that time.

Another way to see that the vertical structure of the envelopes can change is presented by the example of EW Lacertae. We have data on the central depth of the Balmer shell absorption lines which show that the percentage of the stellar disk that is covered by the envelope varies with time. We see the same thing in other Be and shell stars. In 1972 Pleione started with a very thin envelope, which then became more extended vertically, covering more than a stellar diameter. Other stars also show a variation of disk coverage. In this way see that the vertical height of the envelope is an important factor to be considered.

\section{Garrison:}

I am glad that you mentioned some of the higher members of the Balmer series. It seems to me that people have been emphasizing $H \alpha$ to the neglect of the rest of the hydrogen lines, and there is a lot of information there, in particular in the Balmer decrement. I find as a mere observer that the Balmer lines behave quite differently in different Be stars, not only in their decrement but in the width of the emission, whether it turns over into a shell or whether it doesn't turn over into a shell, and these things are not well observed. In addition, many B stars that are considered to be normal B stars have only been observed once. We don't know how many Be stars are lurking in this group, in a quiescent phase. Many Be stars have only been observed once, and we don't know what their behavior will be. Many Be stars have only been observed photometrically once, and we don't know whether they are wildly variable photometrically or whether they flicker with a minute timescale. I would add a timescale of minutes to your timescales of years, months and days.

Plavec:

Anyone else? OK, next speaker, Dr. Bolton, please.

Bolton:

I would like to start out by addressing a broad issue for the observers, and that is what might go into a well designed observing program on Be stars. I think several speakers on the panel, and others at this conference, have eloquently argued about the need for observing any star with as wide a range of techniques over as wide a range of the 
electromagnetic spectrum as possible. The other point that I think has also been made that is very important is that it is necessary to observe these stars over a very long period of time and with relatively dense sampling in time in order to sample the timescales that seem to be involved. This is particularly important, because if the time sampling is not frequent enough, it becomes essentially impossible to separate random variations from periodic or quasi-periodic variations occurring on several timescales. That is a problem that I am afraid is going to plague us for a long time to come. Those two requirements for obtaining understanding, I think, imply that we have to concentrate on a few carefully selected stars, so I would certainly echo Dr. Koubsky's remarks in this regard. I would not look forward to chairing, or perhaps even sitting in a session that would choose those carefully selected stars. I could imagine a real brawl developing, but I think it is very important for us to do that.

In regard to this point, I would like to make what is perhaps a rather archaic statement, and that is to emphasize the continued importance of observing Be stars with photographic spectra. It has become very fashionable to observe with signal-generating detectors, where it is possible to get very high signal-to-noise, and you can see things there that cannot be seen in other ways. But you are throwing away a great deal of information on other lines. Although we did not see many examples, if any, at this meeting, it is possible with careful but not extraordinary care to obtain signal-to-noise of the order of 100 with photographic spectra. There is a tremendous amount of information there that should not be ignored.

There is one other advantage of photographic spectra over digital data, and that is that almost all observatory archives are in the form of photographic plates, whereas much of the digital data is at present not archived. That is going to be a very serious problem, I think, in the long term because given the present state of our ignorance on $\mathrm{Be}$ stars, it is very likely that we are going to want to go back in the future and take another look at these data. If the digital data are not available, either because they are not published in full or they are not archived, we are going to be in rather desperate trouble. In the future we are going to have to repeat a lot of work, and it's going to very time-consuming.

Finally, in this regard I would plead for the observers to publish their complete results including the details of the instrumentation and the techniques used to make whatever measurements they are making. I regret that it is necessary to make such a plea, but when you read the literature you find that it is really common not to include all the necessary information. I would hope that those of us who referee papers would also insist on that, and that those who edit journals would go along with the referees.

Again, given the nature of our near-complete ignorance about Be stars, it is not at all obvious what will be important in the future. To pick an example (not to single anybody out), some of the people who are observing for non-radial pulsations observe both $\mathrm{H} \alpha$ and the $6678 \mathrm{~A}$ He I line. The tendency, however, is to only show or discuss the data for the helium line, because there is emission in the hydrogen line, or the hydrogen line doesn't show much. But there clearly is some importance to those data, and they may be even more important in the future.

Most of the models that we have discussed for the phenomenology that we have seen, in terms of short-term variation in particular, seem to me to be extraordinarily rich. Perhaps many of them are too rich, in a sense that if you fit one feature, it is not at all 
clear what another feature should be doing on the basis of what the model says about the first feature. I think it is very important for those of us who are observers or theoreticians and have particular models in mind to try to narrow down the richness of the models. We should look at individual stars and try to establish consistency over a wide range of features for fitting the model. We should also look at the statistics of the behavior of a number of stars to see which features are important and which aren't.

Balona:

Over the last decade or so, I don't think we have had much progress in understanding the Be phenomenon as such. But thanks to the work of several people on short-period variability, I think we now have a handle to study the underlying star. But before we can do this, I think we must ask the theorists to come up with one or two things. For example, we want to know something about non-radial pulsation in rapidly rotating stars. At present this is a very poorly studied field. Secondly, and most important of all, if we want to interpret these variations in terms of non-radial pulsation, we really need to solve a problem of radiative transfer in a rotating pulsating atmosphere. This is very difficult, but I think it is absolutely essential before we can make much progress, because our current model consists simply of a single layer bobbing up and down on the star, which is totally unrealistic. Also, we need to make progress in mode identification. At the moment, the way this is done is simply by trial and error, and the result is that we have absolutely no idea of how unique the solution is.

As far as observations go, quite clearly we need a strong series of concentrated efforts on just one or two stars, on line profiles, infrared photometry, multi-color photometry, etc. In my opinion, this short-term variability is also prevalent in non-Be stars. I think this must really be decided upon. It is certainly prevalent in Bn stars, and I suspect the 53 Persei stars as well might prove to be manifestations of the same phenomenon, if they are observed regularly enough. So I think there is a lot of work to be done in the future for these short-term variabilities. But I appeal to all observers to please cooperate and concentrate on very few objects; otherwise we will never get to the bottom of this.

Underhill:

I think if one is going to have a survey of observations as recommended by Bolton, and I agree with him, one should try to devise a method of predicting spectra from fairly simple models, but covering a wide range of parameter space. The present methods of modelling are so intensive that one makes one model for one kind of star and that is that. What is basically needed in the future is a "cover the waterfront" method of modelling over a wide range of parameter space, so you can really make sure which part of parameter space we need for stars of type $A$, type $B$, or type $C$ amongst the Be stars.

\section{Doazan:}

I would like just to thank Bolton for emphasizing so heavily the importance of the long-term variations in Be stars. Usually it is extremely difficult to obtain observing time for the long run for a few objects. People think you are just coming to the telescope to look at your favorite star. In fact, what is important is to continue this long time history. We know from Be stars observed over one century that the Be phenomenon is a very longtermed one. It's not just the four-minute or one-week changes that we observe, and this is what we have to understand. 
Bolton:

This is not a field for dilettantes, at least from the observational point of view. You really have to go in for the long term, and it is very important to have homogeneity of observations over a long period of time. The problem of time allocation committees is a difficult one, and perhaps before we make too much further progress that is going to have to be addressed in some way or another.

\section{Harmanec:}

Time is running, and I think we should start to speak about particular stars. The situation is not as bad as you are saying; we do not need to pick only two or three stars. We have two hemispheres, we have summer and winter, so I believe we can pick up to ten objects, and if we pick ten objects we can agree on them. So I propose o Andromedae as one target, for reasons I have explained. For a second object, I propose 88 Herculis. It is a known binary, a $\mathrm{B} 6$ star, again with a very long record, with very many consistent observations. Furthermore, with a B6 star you should not have many problems with emission in the helium line in the red part of the spectrum, so line profile variability could also be investigated. Let's go with other suggestions.

\section{Bolton:}

I really object to that at this stage. I think if we start picking stars right now, we are going with the problem backwards. I think the first thing to do is to try to define problems we want to address, and then pick the stars that are most appropriate for addressing those problems. I agree that we want to pick stars with a long, well-defined history. I also think that at least some of the stars should be ones where the envelope is not a dominant phenomenon, so that we can really look at the photospheres rather than being overwhelmed by the winds. But I don't think we are going to settle these issues in this forum.

\section{Snow:}

I wanted to respond to your comments about archiving of data. I think that is an extremely important point, and historically it has always been difficult. You are right that photographic plates tend to make archiving happen, because plates are material things that people put in drawers and don't lose, and observatories sometimes keep them. I would prefer, however, that you don't blame modern electronic detectors for the difficulty, both in wavelength coverage that you alluded to, and in archiving. After all, the space program is archiving its data rather thoroughly, and in centralized places, and it is almost entirely electronic types of detectors involved. I think your comment about photographic plates was perhaps aimed more at wavelength coverage, because plates very often, especially moderateto low-dispersion plates, tend to provide a lot of wavelength coverage. There is a lot of information there that gets put in those drawers that people can look at later. I think the fact that most people who are now using solid state electronic detectors are only covering small pieces of the spectrum is more a by-product of the types of observations people are trying to do. I think people are trying to do very high-dispersion observations with very high signal-to-noise. They are emphasizing single particular features, and the lack of broader coverage is more a data management problem than anything else. You have so many bits of data to deal with when you are doing very high-dispersion observations. In principle, electronic detectors can cover just as much spectrum as a photographic plate. Much larger 
format panoramic detectors are now being developed, and in a very short time it will be possible to obtain spectra with electronic detectors that cover just as much wavelength and include just as much data. I think the real problem is the archiving problem, and that observatories that are beginning to develop electronic detectors should, in parallel with that, be developing electronic archives and insisting that the observatory is allowed copies of all the data to be kept.

\section{Garrison:}

I think that when you talk about electronic detectors being available, you don't realize that they are available at the big observatories, but the observatories that are likely to have the time to monitor these objects over a long period of time in a stable way are likely to be the small observatories, which don't have the money for electronic detectors. So there we are going to have to rely on some old fashioned techniques.

Snow:

No argument there.

Plavec:

Next speaker, Myron Smith.

Smith:

Unlike many of the other speakers, I am a manifest interloper in the field of Be stars, and I feel frankly a bit cowed at this array of observational data that I see in front of me, that has been considered without definite conclusions emerging, and with the consideration of various astrophysical environments and processes that have to be considered to solve these problems. In a situation such as this, my own personal approach is to take what I think is one of several equally valid avenues, and to break off a very small piece of the problem that I can understand; to start from normal stars and build up in complexity to more pathological cases. In that connection, I should perhaps give the punchline of my remarks here, which is to announce the formation of the kind of observational campaign that Bolton and Balona and Harmanec were just talking about; probably to monitor two or three stars very intensively, in a very catholic way in terms of time coverage and wavelength coverage.

What I would like to do today is to say a few words about something I do understand, or maybe misunderstand the least, and that is line profile variations, but I won't confine my remarks to that. I will confront you with a rather dichotomous badnews/good-news approach. When you start to look at the periodic line profile variations in normal Be stars, what you start to see, especially for the rapid rotators that I think are a relevant comparison here, is a breakdown in the nice uniform periodic behavior that you would expect, whether it's an oblique rotator or a pulsator. The thing that you notice is that the complexities of the departures from periodicity seem to go up with the amplitude. It is probably related to the non-linear aspects of waves in these stars.

However, the observational problem that I want to address is that no two observations a cycle apart are ever seen to exactly repeat. The observations are good enough that we can say that. That's sort of the bad news, because it means that non-simultaneous observations, or rather near-simultaneous observations (a night apart, for example) are not 
good enough. The good-news aspect to this is that if you keep monitoring this, there is potential physical information in those departures, and that is going to help us determine the link between the processes in the normal stars and at least some of the mild Be stars.

The situation seems to be even worse for the Be stars. As John Percy has been telling us, many of them do show periodic photometric variations, but even in those cases there are cycle-to-cycle or night- to-night variations in the amplitudes.

Finally, I think a problem where we are just scratching the surface right now is the superficial lack of direct tracking between the variations in the optical wavelength and the far-UV variations observed by Voyager. Is it true that the monochromatic and the bolometric fluxes don't track each other? If they do, that could tell us something very interesting in terms of physical mechanisms such as whether it is a geometry effect, a temporary radius change in the star, or an effective temperature change.

I brought up the point of the temporal aspects of this yesterday. Spectral transients occur in the form of traveling bumps in the line profiles. This is sort of sad in a way, because it is very complicated, and there is a lot of chaff out there to be separated from the wheat. Even if we think we understand the physical mechanism, we can't predict it. So the sad conclusion is that the problem spectroscopically, even for one small piece of this (such as monitoring the line profile variations), is just too big for one observer.

The good news is that we have some new instrumentation and a lot of energy out there that $I$ think can get at this problem. Simultaneous observations are giving us greater confidence in our results. As recently as one year ago, people expressed skepticism to me about the reality of some of the weak features that we see travelling across the line profiles. Now in some cases we have simultaneous observations from different observatories that confirm the reality of features that are sometimes so weak that I would not have trusted them myself, if they were only seen in one spectrum. These simultaneous observations suggest that if you are going to argue that these weak features can't be trusted, it's going to be more and more of an uphill fight. So, the good news is that the instrumentation is available to get at these kinds of problems, and that the features that we are observing are beginning to be seen as trustworthy.

The other news is that there are a lot of energetic astronomers interested in these problems. There are several of us that congregated in this room yesterday to meet in a sort of exploratory way to plan an international campaign over the next few years on just a few (probably two, three, or four) stars. The kinds of questions that might be addressed would be questions such as those outlined here: the discrimination between pulsation and other models, the separating out of profile components, and finally the precursors in line profile of events that may lead to minor emission outbursts. However, I want to emphasize that this campaign is unique in one aspect, in that it will incorporate multiple-observatory high-dispersion spectroscopy, but it will not be confined to that. To be sure, we need the wavelength coverage, the behavior in different lines, equivalent width variations, and photographic spectroscopy. We desperately need correlations with photometry, both in the visual and ultraviolet, and let's not forget the behavior of the C IV lines that several people here have discussed.

I am going to conclude this by saying this is an announcement of our intent. Should you be interested in participating in this campaign, please contact some of the 
principles who are Diedrich Baade, Tom Bolton, and a small committee that is going to be coordinating the observations consisting of John Percy, Huib Henrichs and myself.

Plavec:

Thank you very much. Before we finish, I would like to ask you to vote on some very simple questions. Make up your mind quickly. Your first choice: do you believe that all Be stars as defined by Collins are just birds of one feather; that is, does one model explain all of them? Second choice: do you believe that there are at least two different groups of Be stars, which require two basically different models? Third choice: do you believe that the complexities are even worse and more desperate, and that at least three models or more will be needed to explain Be stars? OK, who is in favor of one single model that explains every star, modified present model or something improved in the future? I see we have an overwhelming majority: three votes. Who is of the opinion that Be stars are a mixture of two different populations? About 15. Who is of the opinion that things are even worse, and that there are at least three mixtures among what we call the classical Be stars. The vast majority of you. Who doesn't care at all?

\section{Marlborough:}

I won't take too much of your time before we all disperse to the four corners of the earth to get back to work. I don't know how all of you feel but I think I am probably more confused now than I was before, and that may be the same sort of situation that some of you feel you are in also. But, certainly the confusion didn't arise as a result of anything the local organizing committee has done, so I hope you will join me in thanking Ted and his cadre of workers who have made this past week a very enjoyable one.

\section{Snow:}

Thank you; I appreciate that. But I won't let it pass without specifically naming Karen Bjorkman, who coordinated all the students who have been so helpful, and also Cynthia Anderson in the CASA office, who helped many of you with logistical arrangements. I think we owe them both a great debt of thanks. 